\title{
Assimilation Experiment of Lidar Measurements for Wake Turbulence*
}

\author{
Takashi MISAKA**, Takeshi OGASAWARA**, Shigeru OBAYASHI**, \\ Izumi YAMADA $^{* * *}$ and Yoshinori OKUNO ${ }^{\dagger}$ \\ ** Institute of Fluid Science, Tohoku University \\ 2-1-1 Katahira, Aoba-ku, Sendai, Miyagi 980-8577, Japan \\ E-mail: misaka@edge.ifs.tohoku.ac.jp \\ *** Electronic Navigation Research Institute \\ 7-42-23 Jindaiji-higashi-machi, Chofu, Tokyo, 181-0012, Japan \\ $\dagger$ Japan Aerospace Exploration Agency \\ 6-13-1 Osawa, Mitaka, Tokyo 181-0015, Japan
}

\begin{abstract}
Numerical simulation of wake turbulence was performed by integrating the lidar measurements using four-dimensional variational method. A bogus vortex technique was adopted to ensure the existence of wake vortices in the flow field. The validation of the method was performed by an idealized test case using virtual lidar measurement which was produced by the reference simulation of a vortex pair. The results of the validation showed the convergence of a vortex parameter such as a circulation to the parameter of reference simulation case. It was also confirmed that the velocity distribution on a measurement plane agreed with reference one.
\end{abstract}

Key words : Wake Turbulence, Lidar, Four-Dimensional Variational Method

\section{Introduction}

The growth of air traffic causes a lack of airport capacity in major airports. Wake turbulence is one of the factors which restricts the number of takeoff and landing. Since the strength of tip vortices of an aircraft is proportional to the generating lift of the aircraft, the wake turbulence of aircraft that is taking off is strong in particular. The influence of wake turbulence varies depending on surrounding weather conditions, therefore, the present time intervals for takeoff and landing are considered to be long enough for many cases. Meanwhile, unreasonable shortening of aircraft separation would cause an accident due to the wake turbulence. For these reasons, an efficient control of takeoff and landing based on various weather conditions is required.

Numerical simulation of a vortex pair which represents simplified tip vortices of an aircraft has been performed using large eddy simulation (LES) and direct numerical simulation (DNS) for the understanding of wake turbulence. ${ }^{(1)-(3)}$ Although the numerical simulation could reproduce a detailed flow structure of tip vortices, it is difficult to incorporate the effect of actual weather conditions at an airport which significantly affects the decay of the tip vortices. Usually, the atmospheric effect is considered by atmospheric boundary layer ${ }^{(4)}$ and/or homogeneous turbulence generated based on measured spectrum of the atmosphere such as a modified von Karman spectrum. ${ }^{(5),(6)}$ It is well known that the atmospheric turbulence affects the decay of wake vortices.

A light detection and ranging (lidar) is a effective tool to measure the wake turbulence of an operating aircraft. Real-time measurement and visualization are also possible by using the lidar. The lidar measurements of wake turbulence have been conducted at several major airports. In Japan, Electric Navigation Research Institute (ENRI) set a lidar system for wake turbulence measurements at Sendai Airport. ${ }^{(7),(8)}$ A lidar detects aerosol, which is dust and

*Received 18 Jan., 2008 (No. 08-0036) [DOI: 10.1299/jfst.3.512] 
vapor in the atmosphere, as a measurement object, thus it is possible to measure the wind velocity even in a fine day, different from a radar. For the understanding of flow structure of wake turbulence, however, there are some drawbacks such as a lack of spatial resolution in the line of sight (LOS) direction and the difficulty in tracking tip vortices for a long time due to the disturbance in the atmosphere. For the case of a lidar at Sendai Airport, the measurement is obtained every $30[\mathrm{~m}]$ in the LOS direction. This limitation is not desirable because a core radius of wake vortex is in the order of several meters. Reproduction of a detailed structure of wake turbulence is required even if the vortex position is detected by the lidar because the strength and behavior of wake turbulence is not fully explained only by the cross-sectional measurement of the lidar. Therefore, it is worthwhile to clarify the relationship between the three-dimensional structure of wake turbulence and the measured velocity distribution by using the lidar.

The authors have been investigated atmospheric turbulence related to flight of aircraft, such as clear air turbulence ${ }^{(9),(10)}$ and wake turbulence ${ }^{(11),(12)}$ based on measurement-integrated simulation approach. The concept of the measurement-integrated simulation was introduced by Hayase et al. ${ }^{(13)}$ In the present study, the measurement integration was performed based on the data assimilation method used in numerical weather prediction researches, but applied to open bounded, highly unsteady flows such as atmospheric turbulence. In this paper, an assimilation experiment of lidar measurements was performed, which is aiming for the measurement-integrated simulation of aircraft's wake turbulence using the lidar measurements at Sendai Airport. Four-dimensional variational (4D-Var) method was applied to integrate lidar measurements with the three-dimensional computational fluid dynamics (CFD) simulation. A bogus vortex technique was adopted to ensure the existence of wake vortices in the flow field. The assimilation experiment was performed by an idealized test case using virtual lidar measurement which is produced by the reference simulation of a vortex pair.

\section{Methods}

\subsection{Flow Simulation Methods}

Basic equations are the three-dimensional compressible Navier-Stokes equations. Inviscid numerical flux is computed based on Roe's flux difference splitting method using primitive variables interpolated by a higher-order monotonic upwind scheme for conservation laws (MUSCL) scheme. ${ }^{(14)}$ A total-variation-diminishing limiter is not used in the MUSCL interpolation. Viscous term is evaluated by the second-order central difference. Smagorinsky model $\left(C_{s}=0.16\right)$ was used for turbulence closure with the modification for rotational flow. ${ }^{(3)}$ The fourth-order Runge-Kutta method is used for the time integration. The accuracy of the solver was confirmed using Doswell's frontogenesis model, in which time evolution of flow field is specified analytically. ${ }^{(9)}$

\subsection{Assimilation of Lidar Measurements}

The 4D-Var method is employed to integrate the lidar measurement into the numerical simulation. The objective of data assimilation based on the 4D-Var method is to obtain an initial flow condition which reproduces corresponding measurements during the unsteady flow simulation starting from this initial flow condition. ${ }^{(15)}$ The cost function of the 4D-Var is defined based on the difference between lidar measurements and corresponding numerical results. In the present study, the flow variables to be compared to the lidar measurement are obtained by extracting LOS component of velocity vector on a measurement plane shown in Fig. 1. Evaluation of the cost function is performed during the numerical simulation with simulating the lidar measurement process, that is, scanning of laser light in elevator and azimuth angle direction and averaging every 30 [m] in LOS direction. The cost function is expressed as a function of initial flow variables on the measurement plane $\boldsymbol{Q}_{0}$ as follows:

$$
J_{l}\left(\boldsymbol{Q}_{0}\right)=\frac{1}{2} \sum_{i=0}^{N}\left[H_{i}\left(\boldsymbol{Q}_{i}\right)-\boldsymbol{y}_{i}\right]^{T} R_{i}^{-1}\left[H_{i}\left(\boldsymbol{Q}_{i}\right)-\boldsymbol{y}_{i}\right],
$$


where $\boldsymbol{y}_{i}$ is a measurement variable vector, $R_{i}=\left\langle\Delta \boldsymbol{y}_{i}\left(\Delta \boldsymbol{y}_{i}\right)^{T}\right\rangle$ is an observation error covariance matrix and $H_{i}$ is an observation operator which converts the dimension of computational flow variables into that of the measurement vector. Subscript $i$ shows a time step of the flow computation and $N$ is the total number of time steps. Equation (1) is a function of $\boldsymbol{Q}_{0}$, that is, the assimilation problem is defined as the minimization of $J_{l}\left(\boldsymbol{Q}_{0}\right)$ by changing a control parameter $\boldsymbol{Q}_{0}$.

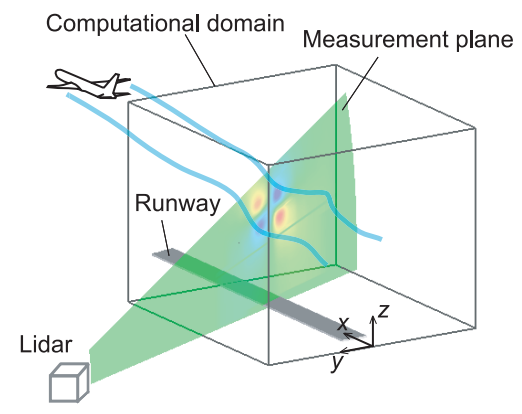

Fig. 1 Schematic of lidar measurement plane and computational domain

Since the lidar measurement consists of a LOS velocity component, there is not enough information to reproduce a velocity profile of wake vortices. To guarantee the vortex structure of a vortex pair in a flow field, 'bogus' vortex technique, which is used to initialize a typhoon in the numerical weather prediction research, ${ }^{(16)}$ was employed. In this technique, a specific vortex profile based on the observation is set in the flow field by modifying the cost function. In the present study, the parameters which determine bogus tip vortices were also considered as the control parameter of the modified cost function so that the strength and position of the vortex pair is determined during a assimilation process. The modified cost function is represented as follows:

$$
\begin{aligned}
& J_{t}\left(\boldsymbol{Q}_{0}, \boldsymbol{P}_{v}, \boldsymbol{P}_{a}\right)=J_{l}\left(\boldsymbol{Q}_{0}\right)+J_{b}\left(\boldsymbol{Q}_{0}, \boldsymbol{P}_{v}, \boldsymbol{P}_{a}\right), \\
& J_{b}\left(\boldsymbol{Q}_{0}, \boldsymbol{P}_{v}, \boldsymbol{P}_{a}\right)=\frac{1}{2}\left[\boldsymbol{Q}_{0}-F_{v}\left(\boldsymbol{P}_{v}\right)-F_{a}\left(\boldsymbol{P}_{a}\right)\right]^{T} B^{-1}\left[\boldsymbol{Q}_{0}-F_{v}\left(\boldsymbol{P}_{v}\right)-F_{a}\left(\boldsymbol{P}_{a}\right)\right],
\end{aligned}
$$

where $F_{v}\left(\boldsymbol{P}_{v}\right)$ represents a specific vortex pair model defined by a parameters $\boldsymbol{P}_{v}$ which consists of a core radius, circulation and vortex position of each vortices. Here, Hallock-Burnham vortex model was assumed as $F_{v}\left(\boldsymbol{P}_{v}\right)$, but an arbitrary vortex model can be applied for $F_{v}\left(\boldsymbol{P}_{v}\right)$. $F_{a}\left(\boldsymbol{P}_{a}\right)$ shows averaged horizontal wind components defined by a parameter $\boldsymbol{P}_{a} . B_{i}$ is an error covariance matrix of a bogus vortex pair. By minimizing the modified cost function $J_{t}\left(\boldsymbol{Q}_{0}, \boldsymbol{P}_{v}, \boldsymbol{P}_{a}\right)$, it is expected to reproduce a vortex structure of wake turbulence while keeping the flexibility of velocity field representation. The modified cost function $J_{t}\left(\boldsymbol{Q}_{0}, \boldsymbol{P}_{v}, \boldsymbol{P}_{a}\right)$ is not well-defined because the control parameters $\boldsymbol{Q}_{0}, \boldsymbol{P}_{v}$ and $\boldsymbol{P}_{a}$ depend on each other. To avoid this, the control parameter $\boldsymbol{Q}_{0}$ is redefined as,

$$
\boldsymbol{Q}_{0}=\Delta \boldsymbol{Q}_{0}+F_{v}\left(\boldsymbol{P}_{v}\right)+F_{a}\left(\boldsymbol{P}_{a}\right),
$$

therefore, Eq. (2) becomes as,

$$
\begin{aligned}
J_{t}\left(\Delta \boldsymbol{Q}_{0}, \boldsymbol{P}_{v}, \boldsymbol{P}_{a}\right)= & J_{l}\left(\Delta \boldsymbol{Q}_{0}, \boldsymbol{P}_{v}, \boldsymbol{P}_{a}\right)+J_{b}\left(\Delta \boldsymbol{Q}_{0}\right) \\
= & \frac{1}{2}\left[H_{0}\left[\Delta \boldsymbol{Q}_{0}+F_{v}\left(\boldsymbol{P}_{v}\right)+F_{a}\left(\boldsymbol{P}_{a}\right)\right]-\boldsymbol{y}_{0}\right]^{T} R_{0}^{-1}\left[H_{0}\left[\Delta \boldsymbol{Q}_{0}+F_{v}\left(\boldsymbol{P}_{v}\right)+F_{a}\left(\boldsymbol{P}_{a}\right)\right]-\boldsymbol{y}_{0}\right] \\
& +\frac{1}{2} \sum_{i=1}^{N}\left[H_{i}\left(\boldsymbol{Q}_{i}\right)-\boldsymbol{y}_{i}\right]^{T} R_{i}^{-1}\left[H_{i}\left(\boldsymbol{Q}_{i}\right)-\boldsymbol{y}_{i}\right] \\
& +\frac{1}{2} \Delta \boldsymbol{Q}_{0}^{T} B^{-1} \Delta \boldsymbol{Q}_{0} .
\end{aligned}
$$

$J_{t}\left(\Delta \boldsymbol{Q}_{0}, \boldsymbol{P}_{v}, \boldsymbol{P}_{a}\right)$ is minimized by the gradient based method. Equations to obtain the gradient are derived as follows:

$\lambda_{N+1}=0$, 


$$
\begin{aligned}
& \lambda_{i}=M_{i}^{A} \lambda_{i+1}+H_{i}^{A} R_{i}^{-1}\left[H_{i}\left(\boldsymbol{Q}_{i}\right)-\boldsymbol{y}_{i}\right], \quad(i=N, N-1, \cdots, 0), \\
& \nabla_{\Delta Q_{0}} J_{t}=\lambda_{0}+B^{-1} \Delta Q_{0} \\
& \nabla_{P_{v}} J_{t}=F_{v}^{A} \lambda_{0} \\
& \nabla_{P_{a}} J_{t}=F_{a}^{A} \lambda_{0}
\end{aligned}
$$

where $\lambda_{i}$ is an adjoint vector, $M_{i}^{A}$ is an adjoint operator of Navier-Stokes equation, $H_{i}^{A}$ is an adjoint operator of $H_{i}$. The derivation of these equations based on variational method can be found in papers by authors. ${ }^{(10),(12)}$ Equations (6) to (8) show that the gradient of $J_{t}$ is obtained by the inverse time integration of the adjoint equation with a force term: $H_{i}^{A} R_{i}^{-1}\left[H_{i}\left(\boldsymbol{Q}_{i}\right)-\boldsymbol{y}_{i}\right]$. An adjoint operator $M_{i}^{A}$ in Eq. (7) is obtained by linearizing a Navier-Stokes code directly. A measurement operator $H_{i}$ and its adjoint operator $H_{i}^{A}$ correspond to the procedure to obtain lidar measurements from a simulated flow field and its adjoint operation, respectively. $F_{v}{ }^{A}$ and $F_{a}^{A}$ are adjoint operators of $F_{v}$ and $F_{a}$, respectively. Using the obtained gradient, the minimization of $J_{t}$ is performed by the quasi-Newton method. Hessian matrix is approximated using the Broyden-Fletcher-Goldfarb-Shanno method. Based on the updated initial flow variables, the numerical simulation and the evaluation of $J_{t}$ are conducted again. The above procedures are repeated until the objective function converges. Both Navier-Stokes and its adjoint code are parallelized using OpenMP.

\subsection{Computational Setup}

An assimilation experiment was set by a reference simulation of a vortex pair with simulating actual measurement process in the numerical simulation to obtain the virtual lidar measurements. Hallock-Burnham vortex model was used for an initial condition of the reference simulation. Homogeneous turbulence and averaged wind components were not considered for the assimilation experiments. And, matrices $R_{i}$ and $B$ were set to a unit matrix. Here, one measurement plane is considered, therefore, the velocity profile on the measurement plane is swept in $x$-axis direction in Fig. 1 to obtain a three-dimensional vortex pair.

\section{Results of Assimilation Experiment}

Figure 2 shows histories of cost functions, $J_{t}, J_{l}$ and $J_{b}$, during the iteration of 4D-Var method. Vertical axis shows the value of cost functions and horizontal axis shows iteration step. As a flow field is modified based on lidar measurements, the value of $J_{b}$ increases because the modified flow field initially does not agree with the velocity profile represented by a vortex model. After the value of $J_{l}$ becomes smaller than $J_{b}$, however, $J_{b}$ decreases by modifying the parameters of a vortex model.

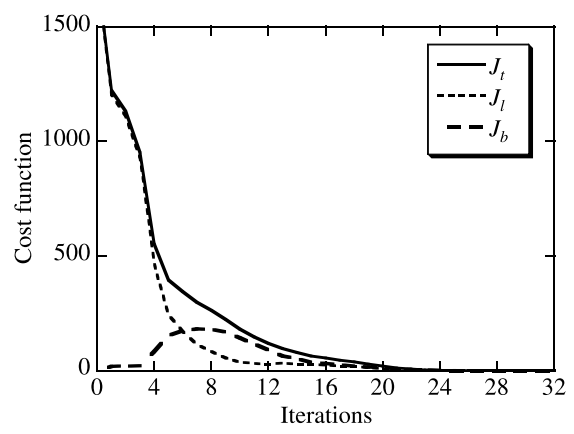

Fig. 2 History of cost functions

Figure 3 shows the convergence history of vortex circulation. Vertical axis shows the circulation which is calculated by the line integral along closed circle around a vortex center and averaged between 5 to $15[\mathrm{~m}]$ of radius; $\Gamma_{5-15}$, and horizontal axis shows iteration steps 
of 4D-Var method. Since the assimilation starts without initial velocity distribution, the circulation increases from zero. The circulation becomes almost identical value at 32 nd iteration compared to the reference one. Figure 4 shows the radial profile of circulation obtained from an initial flow state at several iteration steps. From this results, it is confirmed that the radial profile of the vortex converges to the reference one, not only the integral quantity such as $\Gamma_{5-15}$.

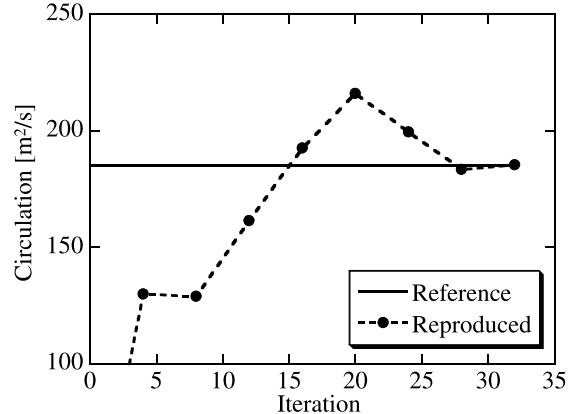

Fig. 3 History of initial vortex circulation during the iteration of 4D-Var method

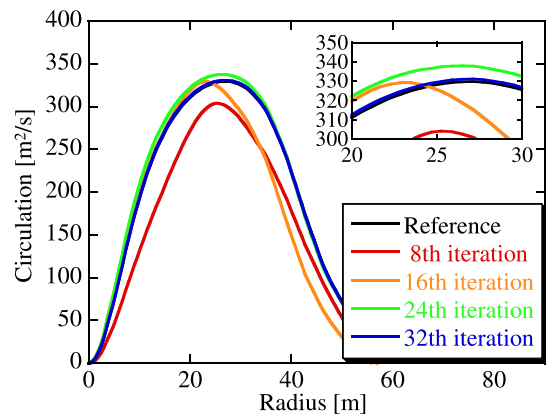

Fig. 4 Radial profile of initial circulation at several iteration steps

Figure 5 shows the comparison of the velocity distribution on measurement plane. Figure 5a is LOS component of velocity extracted from the reference simulation of a vortex pair. And Fig. 5b to $5 \mathrm{~d}$ show the velocity distribution at 1 st, 16 th and 32 nd iteration, respectively. At 16th iteration, the most part of velocity distribution is recovered, however, there is some fluctuations of velocity distribution compared to the reference one. These fluctuations result in the discrepancy of radial circulation profile over 20 [m] of core radius in Fig. 4.
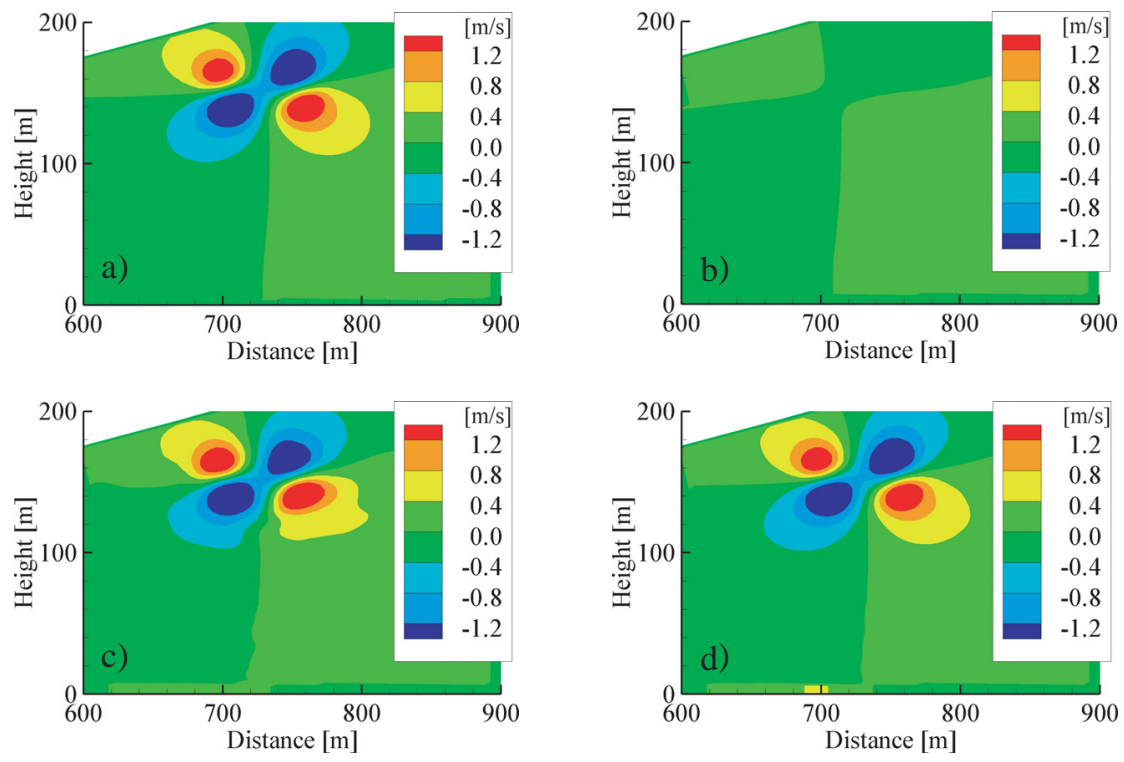

Fig. 5 Comparison of velocity distribution on measurement plane, a) reference, b) 1st iteration, c) 16th iteration, d) 32nd iteration

Figure 6 shows three-dimensional structure of a vortex pair visualized by iso-vorticity surface. It is confirmed that the strength of a vortex pair becomes larger as the iteration step increases. In addition, a vertical position of the vortex pair at 32 nd iteration, it can be confirmed by $z$-axis in these figures, is higher than that of 1 st iteration. Since the vortex pair moves downward due to their own circulation, the position measured by lidar is lower than its initial position. As the circulation of the vortex pair increases, the vertical position of the vortex pair moves upward because the movement on the vortex pair becomes larger as the circulation increases. It is possible to reproduce the initial position of a vortex pair 
a)

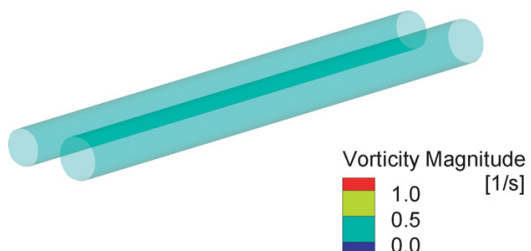

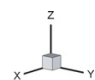

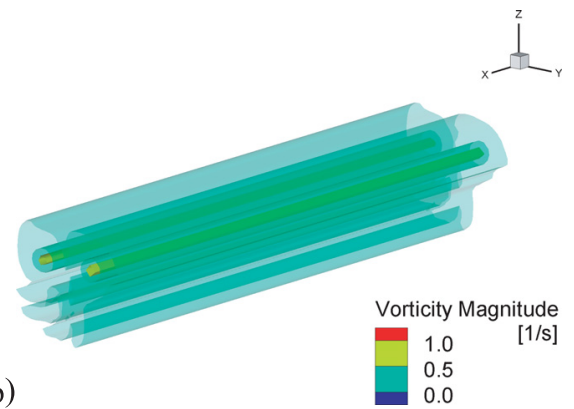

b)
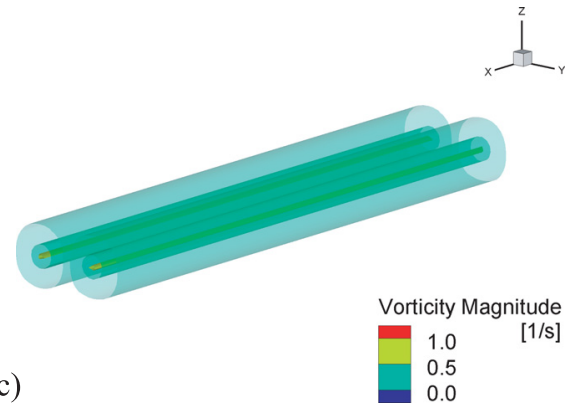

Fig. 6 Structure of a vortex pair visualized by iso-vorticity surface at several iteration step, a) 1st iteration, b) 16th iteration, c) 32nd iteration

not only the strength by the use of 4D-Var method, which can consider the time accuracy of measurements.

\section{Conclusions}

Assimilation experiment of lidar measurement was performed, which is aiming for the reproduction of three-dimensional flow structure of wake turbulence at an airport. The 4DVar method with bogus vortex technique was formulated in which the parameters of the bogus vortex was also treated as control parameters. The validation of the method was performed by using virtual lidar measurement obtained from a numerical simulation of a vortex pair with simulating the lidar measurement process. Results of the assimilation experiment showed the reproducibility of an original vortex pair. The agreement was also confirmed by the circulation evaluated directly from the flow field.

\section{References}

( 1 ) WakeNet2-Europe website: http://www-mip.onera.fr/projets/wakenet2-europe/.

( 2 ) Garten, J. F., Werne, J., Fritts, D. C. and Arendt, S. Direct Numerical Simulations of the Crow Instability and Subsequent Vortex Reconnection in a Stratified Fluid, Journal of Fluid Mechanics, Vol. 426, 2001, pp. 1-45.

( 3 ) Proctor, F. H., Hamilton, D. W. and Han, J. Wake Vortex Transport and Decay in Ground Effect: Vortex Linking with the Ground, AIAA Paper 2000-757, 2000.

( 4 ) Holzäpfel, F., Gerz, T., Frech, M. and Dornbrack, A. Wake Vortices in Convective Boundary Layer and Their Influence on Following Aircraft, Journal of Aircraft, Vol. 37, No. 6, 2000, pp. 1001-1007.

( 5 ) Risso, F., Corjon, A. and Stoessel, A. Direct Numerical Simulations of Wake Vortices in Intense Homogeneous Turbulence, AIAA Journal, Vol. 35, No. 6, 1997, pp. 1030-1040.

( 6 ) Bechara, W., Bailly, C., Lafon, P. and Candel, S. M. Stochastic Approach to Noise Modeling for Free Turbulent Flows, AIAA Journal, Vol. 32, No. 3, 1994, pp. 455-463.

( 7 ) Kaku, N. Observation of Wake Turbulence using Lidar, IEICE Technical Report, SSS2006-17, 2006 (in Japanese).

( 8 ) Matsuda, T., Ando, T., Furuta, M., Hirano, Y., Nakayama, T. and Komiyama, S. UpperAir Wind Observation with an All-Fiber Coherent Doppler Lidar System (Comparison 
of Ultrasonic Wind Measurement Sensor), Digest of 24th Laser Sensing Symposium, 2005 (in Japanese).

( 9 ) Misaka, T., Obayashi, S. and Endo, E., Flow Field Reproduction Based on Flight Data Using Numerical Simulation, JSFM 19th Computational Fluid Dynamics Symposium, C5-1, 2005 (in Japanese).

(10) Misaka, T., Obayashi, S. and Endo, E. Numerical Simulation of Clear Air Turbulence Using 4D-Var, 45th AIAA Aerospace Sciences Meeting and Exhibit, AIAA Paper 200781, 2007.

(11) Misaka, T., Obayashi, S., Kaku, E. and Okuno, Y., Measurement-Integrated Simulation of Wake Turbulence, JSFM 20th Computational Fluid Dynamics Symposium, D7-2, 2006 (in Japanese).

(12) Misaka, T., Ogasawara, T., Obayashi, S., Yamada, I. and Okuno, Y., MeasurementIntegrated Simulation of Wake Turbulence, 46th AIAA Aerospace Sciences Meeting and Exhibit, AIAA Paper 2008-466, 2008.

(13) Hayase, T. and Nisugi, K. and Shirai, A., Numerical Realization for Analysis of Real Flows by Integrating Computation and Measurement, International Journal for Numerical Methods in Fluids, Vol. 47, No. 6-7, 2005, pp. 543-559.

(14) Yamamoto, S. and Daiguji, H. Higher-Order-Accurate Upwind Schemes for Solving the Compressible Euler and Navier-Stokes Equations, Computer and Fluids, Vol. 22, No. 2/3, 1993, pp. 259-270.

(15) Kalnay, E. Atmospheric Modeling, Data Assimilation and Predictability, Cambridge University Press, 2003.

(16) $\mathrm{Pu}, \mathrm{Z}$. and Braun, S. A. Evaluation of Bogus Vortex Techniques with Four-dimensional Variational Data Assimilation, Monthly Weather Review, Vol. 129, 2001, pp. 2023-2039. 